\title{
A REFERENCE TRAJECTORY BASED DISCRETE TIME SLIDING MODE CONTROL STRATEGY
}

\author{
ANDRZEJ BARTOSZEWICZ ${ }^{a, *}$, KATARZYNA ADAMIAK $^{a}$ \\ ${ }^{a}$ Institute of Automatic Control \\ Łódź University of Technology, Stefanowskiego 18/22, 90-924 Łódź, Poland \\ e-mail: andrzej.bartoszewicz@p.lodz.pl
}

\begin{abstract}
This study presents a new, reference trajectory based sliding mode control strategy for disturbed discrete time dynamical systems. The desired trajectory, which is generated externally according to an existing switching type reaching law, determines the properties of the emerging sliding motion of the system. It is proved that an appropriate choice of the trajectory generator parameters ensures the existence of the quasi-sliding motion of the system according to the definition by Gao et al. (1995) in spite of the influence of disturbances. Moreover, the paper shows that the application of the desired trajectory based reaching law results in a significant reduction in the quasi-sliding mode band width and errors of all state variables. Therefore, in comparison with Gao's control method, the system's robustness is increased. The paper also presents an additional modification of the reaching law, which guarantees a further reduction in the quasi-sliding mode band in the case of slowly varying disturbances. The results are confirmed with a simulation example.
\end{abstract}

Keywords: discrete time systems, sliding mode control, reaching law, reference trajectory.

\section{Introduction}

Sliding mode control is one of the most attractive strategies among modern control methods. Due to its high computational efficiency and low implementation costs, it has recently become very popular. However, the idea of variable structure systems has been present in control theory for decades. First sliding mode systems were studied in the Soviet Union in the early 1950s by Emelyanov (1957; 1964), Utkin (1977; 1978; 1984) and Itkis (1976). They proposed to use a discontinuous state-feedback based control law in order to adjust the structure of the system according to its state during the control process. Namely, a sliding mode controller switches the structure of the system along an arbitrarily chosen sliding surface, which ensures stable steady state behaviour. Therefore, the dimensions of the dynamical problem are reduced by the number of independent inputs, and the system becomes highly insensitive to external disturbances and parameter uncertainties.

The invariance conditions were studied in detail by Draženović (1969), and later on multiple authors elaborated on sliding mode control for continuous time

*Corresponding author systems (DeCarlo et al., 1988; Hung et al., 1993; Edwards and Spurgeon, 1998; Sabanovic, 2011). However, the implementation of digital controllers was one of the factors that triggered the chattering phenomenon. As in discrete time the control signal cannot be changed smoothly, the systems tend to oscillate around the switching plane (Zinober et al., 1982; Chang et al., 1990; Bartolini et al., 1998; 2000). Profound analysis of the problem drew the attention to discrete time dynamical systems. At the end of the 20th century, Milosavljević (1985) for the first time defined the conditions of the existence of sliding motion in discrete time systems. His work was followed by numerous authors, who analyzed the stability of discrete time sliding modes (Sarpturk et al., 1987; Kotta et al., 1989; Utkin and Drakunov, 1989; Furuta, 1990). A breakthrough occurred when Gao et al. (1995) defined the quasi-sliding motion as follows:

- The representative point of the system moves monotonically, in the so-called reaching phase, from any initial position towards the sliding surface and crosses it in finite time.

- Once the representative point of the system has crossed the sliding hyperplane for the first time, 
a sliding phase begins, where the sliding plane is recrossed and the sign of the sliding variable changes in each following step.

- In the sliding phase the representative point of the system remains in an a priori known vicinity of the sliding surface.

The authors also introduced a reaching law approach. Their idea assumes selecting a sliding hyperplane (Luis-Delgado et al., 2017), which ensures stable steady state behaviour, and describing the sliding variable with a function, which drives the representative point to the chosen plane. Several researchers have worked to improve Gao's control strategy (Bartoszewicz, 1996; Ren et al., 2013; Veselic et al., 2010; Qu et al., 2014) and numerous new reaching laws have been proposed (Bartoszewicz,1998; Leśniewski and Bartoszewicz, 2015; Bartoszewicz and Leśniewski, 2016; Latosiński, 2017; Latosiński and Bartoszewicz, 2018; Monsees and Sherpen, 2001; Vivekanandan et al., 2008; Golo and Milosavljewic̀, 2000; Niu et al., 2010; Ma et al., 2017; Zhang, 2016; Chakrabarty and Bandyopadhyay, 2015; 2016; Chakrabarty and Bartoszewicz 2016) ever since.

In our work we propose a new, reference trajectory following sliding mode control strategy. We begin with generating a desired evolution of the sliding variable with an existing inverse tangent switching type reaching law of Leśniewski and Bartoszewicz (2015), satisfying the sliding mode conditions defined by Gao et al. (1995). Next, we introduce a new trajectory following reaching law for the real disturbed plant. We prove that our strategy guarantees the existence of the sliding mode as defined by Gao et al. (1995). Moreover, the implementation of the reference trajectory method results in a reduction in the influence of external disturbances and parameter uncertainties on the system, and therefore offers an improvement of its robustness. Then our strategy is modified to ensure a further reduction in the width of the ultimate band in the case of slowly varying disturbance. Lastly, our results are verified with a simulation example and compared with the performance of the original strategy of Gao et al. (1995).

\section{Reference trajectory based SMC strategy}

2.1. Problem statement. In this paper we take into account a discrete time disturbed plant, described by the following state equation:

$$
\boldsymbol{x}(k+1)=\boldsymbol{A} \boldsymbol{x}(k)+\boldsymbol{b} u(k)+\boldsymbol{b} d(k),
$$

where $x(k)$ is an $n \times 1$ state vector, $\boldsymbol{A}$ is the plant's state matrix, $\boldsymbol{b}$ is the input distribution vector, $u(k)$ denotes the control signal and $d(k)$ is a scalar disturbance. The disturbance is bounded by known values: $d_{\min }$ and $d_{\text {max }}$. We aim to drive the system from the initial state, represented by $x_{0}=\boldsymbol{x}(0)$, to the demand state $\boldsymbol{x}_{d}$. Therefore, the error vector in each control step is defined as $\boldsymbol{e}(k)=\boldsymbol{x}_{d}-\boldsymbol{x}(k)$. We introduce the sliding variable $s(k)=\boldsymbol{c e}(k)$, where $\boldsymbol{c}$ is an $1 \times n$ vector selected so that $(\boldsymbol{c} b)^{-1} \neq 0$, and the sliding hyperplane

$$
s(k)=0 .
$$

We denote here by $D(k)=\boldsymbol{c b d}(k)$ the impact of the disturbance on the sliding variable. Moreover, we denote by $D_{1}$ the mean value of $D(k)$ and by $D_{2}$ maximum deviation of $D(k)$ from the mean. We have

$$
\begin{aligned}
& D_{1}=\frac{\boldsymbol{c} \boldsymbol{b} d_{\max }+\boldsymbol{c} \boldsymbol{b} d_{\min }}{2} \\
& D_{2}=\frac{\left|\boldsymbol{c} \boldsymbol{b} d_{\max }-\boldsymbol{c} \boldsymbol{b} d_{\min }\right|}{2} .
\end{aligned}
$$

Having introduced the necessary notation, in the next section we will present how to obtain the reference trajectory for the nominal system (1).

2.2. Reference trajectory. Our idea is to use an existing switching type reaching law to generate the desired evolution of the sliding variable. At the stage of designing the reference trajectory, neither disturbances nor parameter uncertainties are considered. Therefore, we use an inverse tangent reaching law (Leśniewski and Bartoszewicz, 2015), which ensures all the properties of the quasi-sliding mode according to Gao's definition and provides a relatively small width of the ultimate band. We denote by $s_{d}(k)$ the desired sliding variable and set its initial value according to the initial conditions of the plant:

$$
s_{d}(0)=s(0)=\boldsymbol{c}\left(\boldsymbol{x}_{d}-\boldsymbol{x}_{0}\right) .
$$

The reference sliding variable $s_{d}(k)$ must converge to a predefined vicinity of zero. Therefore, in the trajectory generator we use the inverse tangent reaching law (Leśniewski and Bartoszewicz, 2015) in the following form:

$$
s_{d}(k+1)=s_{d}(k)-g \arctan \left[\frac{s_{d}(k)}{g}\right]-\varepsilon \operatorname{sgn}\left[s_{d}(k)\right],
$$

where $g$ and $\varepsilon$ are positive real constants. We define the signum function as

$$
\operatorname{sgn}(z)= \begin{cases}1 & \text { for } z \geq 0 \\ -1 & \text { for } z<0\end{cases}
$$

As the inverse tangent function is bounded, the maximum and the minimum rate of change of the desired sliding variable are limited. Directly from (5) we may write that

$$
\left|s_{d}(k)-s_{d}(k+1)\right|=\varepsilon+g \arctan \left[\frac{s_{d}(k)}{g}\right] .
$$


Consequently, the rate of change of the desired sliding variable is limited as follows:

$$
\varepsilon<\left|s_{d}(k)-s_{d}(k+1)\right|<\varepsilon+g \frac{\pi}{2} .
$$

This proves that the desired sliding variable will change its sign in finite time. We define $k_{0 d}$ as the last moment before the desired trajectory crosses the sliding plane, so the following equality holds:

$$
\operatorname{sgn}\left[s_{d}\left(k_{0 d}\right)\right]=-\operatorname{sgn}\left[s_{d}\left(k_{0 d}+1\right)\right] .
$$

Next, using (5), we express $s_{d}(k+1)$ as

$$
\begin{aligned}
& \operatorname{sgn}\left[s_{d}(k+1)\right]\left|s_{d}(k+1)\right| \\
& =\operatorname{sgn}\left[s_{d}(k)\right]\left\{\left|s_{d}(k)\right|-g \arctan \left[\frac{\left|s_{d}(k)\right|}{g}\right]-\varepsilon\right\} .
\end{aligned}
$$

Therefore, using (9) and (10), we can express the absolute value of $s_{d}\left(k_{0 d}+1\right)$ as follows:

$\left|s_{d}\left(k_{0 d}+1\right)\right|=-\left|s_{d}\left(k_{0 d}\right)\right|+g \arctan \left[\frac{\left|s_{d}\left(k_{0 d}\right)\right|}{g}\right]+\varepsilon$.

It is easy to notice that, for any $\left|s_{d}(k)\right|$,

$$
g \arctan \left[\frac{\left|s_{d}(k)\right|}{g}\right]-\left|s_{d}(k)\right| \leq 0 .
$$

Therefore, from (11) and (12) we conclude that

$$
\left|s_{d}\left(k_{0 d}+1\right)\right| \leq \varepsilon .
$$

For the next time step we may write

$$
\begin{aligned}
& \operatorname{sgn}\left[s_{d}\left(k_{0 d}+2\right)\right]\left|s_{d}\left(k_{0 d}+2\right)\right| \\
& =\operatorname{sgn}\left[s_{d}\left(k_{0 d}+1\right)\right] \\
& \quad \cdot\left\{s_{d}\left(k_{0 d}+1\right)-g \arctan \left[\frac{\left|s_{d}\left(k_{0 d}+1\right)\right|}{g}\right]-\varepsilon\right\} .
\end{aligned}
$$

As the term in curly brackets is always negative, the change in the sign of the desired sliding variable occurs again. Moreover, from (12) and (14) it can be noticed that

$$
\left|s_{d}\left(k_{0 d}+2\right)\right| \leq \varepsilon .
$$

Following the same reasoning for the next control steps, we conclude that, for any $k \geq k_{0 d}$

$$
\operatorname{sgn}\left[s_{d}(k)\right]=-\operatorname{sgn}\left[s_{d}(k+1)\right]
$$

and the absolute value of the desired sliding variable is bounded by

$$
\left|s_{d}(k+1)\right| \leq \varepsilon \text {. }
$$

As shown above, the desired trajectory satisfies the properties of the quasi-sliding mode defined by Gao et al (1995). In the sliding phase the sliding variable is confined to the $\varepsilon$ vicinity of the sliding surface and changes its sign in each consecutive time instant. In the next section, the reference trajectory will be used for the control of a real disturbed plant.
2.3. Reaching law-based control strategy. Now, we will introduce a reference trajectory based reaching law for the system (1):

$$
s(k+1)=s_{d}(k+1)-D(k)+D_{1} .
$$

The reaching law drives the plant's sliding variable in step $k+1$ to its desired value with the accuracy of the disturbance $D(k)$ and compensates for the mean disturbance $D_{1}$. As the desired trajectory is not affected by any perturbation, the plant's performance at time $k+1$ is only influenced by the disturbance from one control step $k$, whereas, in the previous sliding mode control strategies (e.g., Gao et al., 1995; Veselic et al., 2010; Leśniewski and Bartoszewicz, 2015; Golo and Milosavljević, 2000; Monsees and Sherpen, 2001), the sliding variable in each step bears the influence of all the disturbance values from the beginning of the control process. Consequently, implementation of the trajectory following the reaching law improves the system robustness.

Considering (1), (2) and (18), we get the following control signal:

$$
u(k)=(\boldsymbol{c} \boldsymbol{b})^{-1}\left[\boldsymbol{c} \boldsymbol{x}_{d}-\boldsymbol{c A x}(k)-s_{d}(k+1)-D_{1}\right] .
$$

However, following the reference trajectory by the real plant, subject to external disturbance and parameter uncertainties, may be insufficient for the quasi-sliding mode to emerge as the disturbance $D(k)$ may push the system's representative point away from the sliding plane. Therefore, we will provide a certain selection of control parameters $g$ and $\varepsilon$ of the reference trajectory generator that guarantee the sliding motion. Assume that at some instant the sliding variable of the plant changes its sign and denote by $k_{0}$ the last moment before the first change in the sign so that

$$
\begin{aligned}
\operatorname{sgn}[s(0)] & =\operatorname{sgn}[s(1)]=\ldots \\
& =\operatorname{sgn}\left[s\left(k_{0}\right)\right]=-\operatorname{sgn}\left[s\left(k_{0}+1\right)\right] .
\end{aligned}
$$

Further in the paper we will demonstrate that a finite $k_{0}$ actually exists. According to Gao's definition, for the quasi-sliding mode to emerge, the sliding variable must satisfy

$$
\operatorname{sgn}[s(k+1)]=-\operatorname{sgn}[s(k)]
$$

for any $k \geq k_{0}$.

Theorem 1. If $g>2 D_{2} / \pi$ and $\varepsilon>g \tan \left(D_{2} / g\right)$, then the plant's trajectory crosses the sliding plane not later than between steps $k_{0}=k_{0 d}+2$ and $k_{0}+1$. For any $k \geq k_{0}$, (21) is satisfied and the quasi-sliding mode as defined by Gao et al. (1995) emerges. Moreover, for any $k \geq k_{0}+1$ the absolute value of the plant's sliding variable is bounded by $\varepsilon+D_{2}$. 
Proof. We first present $s(k+1)$ according to the reaching laws (18) and (5):

$$
\begin{aligned}
\operatorname{sgn}[s(k+1)]|s(k+1)| & \\
= & \operatorname{sgn}\left[s_{d}(k)\right]\left\{\left|s_{d}(k)\right|-g \arctan \left[\frac{\left|s_{d}(k)\right|}{g}\right]-\varepsilon\right\} \\
& -D(k)+D_{1} .
\end{aligned}
$$

In Section 2.2 it is shown that the desired trajectory crosses the sliding plane for the first time between moments $k_{0 d}$ and $k_{0 d}+1$. Afterwards, for any $k \geq k_{0 d}+1$, the sign of $s_{d}$ changes and its absolute value is upper bounded by $\varepsilon$. Therefore, the term in the curly brackets in (22) is always negative. Moreover, since $D(k)=\boldsymbol{c} \boldsymbol{b} d(k)$ and $d(k)$ is lower and upper bounded by $d_{\min }$ and $d_{\max }$, from (3) we conclude that $\left|-D(k)+D_{1}\right| \leq D_{2}$. Consequently, if

$$
g \arctan \left(\frac{\varepsilon}{g}\right)>D_{2},
$$

then, for any $k \geq k_{0 d}+2$,

$$
\operatorname{sgn}[s(k+1)]=-\operatorname{sgn}\left[s_{d}(k)\right]=\operatorname{sgn}\left[s_{d}(k+1)\right] .
$$

We conclude that a finite $k_{0}$ exists and 21) holds for any $k \geq k_{0}=k_{0 d}+2$ if the reference trajectory parameters are chosen as

$$
g>\frac{2 D_{2}}{\pi}
$$

and

$$
\varepsilon>\tan \left(\frac{D_{2}}{g}\right) .
$$

Next, we may use the reaching law (18) to obtain the quasi-sliding mode band width. As has already been proved, $s_{d}(k)$ satisfies (17) for any $k \geq k_{0 d}+1$. Consequently, in the sliding phase the absolute value of the plant's sliding variable satisfies

$$
|s(k)| \leq \varepsilon+D_{2},
$$

which ends the proof.

The benefits of the reference trajectory based control strategy may be demonstrated by a comparison with the fundamental strategy of Gao et al. (1995). They proved that the implementation of their reaching law results in the ultimate band described by

$$
|s(k)| \leq \varepsilon+2 D_{2},
$$

which is strictly greater than the band (27) achieved in our control method. Moreover, the reaching law used in the reference trajectory generator determined the maximum rate of change in the desired sliding variable in the reaching phase. This feature allows us to restrict the maximum rate of change of the plant's sliding variable as well. We describe the change in $s(k)$ as

$$
\begin{aligned}
s(k)- & s(k+1) \\
= & s_{d}(k)-D(k-1)+D_{1} \\
& -s_{d}(k+1)+D(k)-D_{1} .
\end{aligned}
$$

Considering (8) and (29), we conclude that the maximum absolute rate of change in $s$ is upper bounded by

$$
|s(k)-s(k+1)| \leq \varepsilon+g \frac{\pi}{2}+2 D_{2} .
$$

We may also notice that our control strategy allows the designer to easily adjust the pace of convergence of the system by the choice of parameter $g$ and the width of the ultimate band with the choice of $\varepsilon$.

It has already been noticed by Latosiński and Bartoszewicz (2018) that a certain choice of the control vector $\boldsymbol{c}$ also allows determining the maximum errors of all the state variables in the sliding phase. Assume that the control vector is chosen to place all the poles of the closed-loop system at the origin of the complex plane. Hence the characteristic polynomial satisfies

$$
M(z)=\operatorname{det}\left(\mathbf{1} z-A_{\boldsymbol{c}}\right)=z^{n},
$$

where $A_{\boldsymbol{c}}=\left[\mathbf{1}-\boldsymbol{b}(\boldsymbol{c b})^{-1} \boldsymbol{c}\right] \boldsymbol{A}$ denotes the state matrix of the closed-loop system and $n$ is the system order. In this case, after Latosiński and Bartoszewicz (2018), we conclude that the state vector for any $k \geq k_{0}+n$ in the sliding phase may be expressed as

$$
\boldsymbol{x}(k)=\sum_{l=0}^{n-1} \boldsymbol{A}_{\boldsymbol{c}}^{l} \boldsymbol{b}(\boldsymbol{c b})^{-1} s(k-l) .
$$

The maximum absolute value of the $i$-th state variable in the sliding phase may be determined by multiplying both the sides of (32) by a row vector:

$$
\boldsymbol{v}_{i}=[\underbrace{0 \ldots 0}_{i-1} 1 \underbrace{0 \ldots 0}_{n-i}]
$$

and substituting the maximum absolute values of the sliding variable for $s(k-l)$. Therefore, for Gao's control strategy, the maximum value of the $i$-th state variable in the sliding phase satisfies

$$
\left|x_{i}(k)\right| \leq\left|\left(\varepsilon+2 D_{2}\right) \boldsymbol{v}_{i} \sum_{l=0}^{n-1} \boldsymbol{A}_{\boldsymbol{c}}{ }^{l} \boldsymbol{b}(\boldsymbol{c} \boldsymbol{b})^{-1}\right|,
$$

whereas for the trajectory following strategy it satisfies

$$
\left|x_{i}(k)\right| \leq\left|\left(\varepsilon+D_{2}\right) \boldsymbol{v}_{i} \sum_{l=0}^{n-1} \boldsymbol{A}_{\boldsymbol{c}}{ }^{l} \boldsymbol{b}(\boldsymbol{c} \boldsymbol{b})^{-1}\right| .
$$

From the comparison of (34) and (35) it is clear that the new trajectory following control method reduces the maximum errors of all the state variables. 
2.4. Modified strategy. In this section we consider slowly varying disturbances. We assume that for any $k \geq 0$, the disturbance satisfies

$$
|D(k+1)-D(k)| \leq \Delta<D_{2} .
$$

In that case the reference trajectory based reaching law may be modified, as shown by Bartoszewicz (1998), in order to additionally reduce the width of the ultimate band. The idea is to partially compensate for $D(k)$ using the system's history. Therefore, we modify our reaching law to the following form:

$$
\begin{aligned}
s(k+1)= & s_{d}(k+1)-D(k)+D_{1} \\
& -\sum_{l=0}^{k}\left[s(l)-s_{d}(k)\right] .
\end{aligned}
$$

Considering (1), (2) and (37), we obtain the following control signal:

$$
\begin{array}{r}
u(k)=(\boldsymbol{c b})^{-1}\left\{\boldsymbol{c} \boldsymbol{x}_{d}-\boldsymbol{c A} \boldsymbol{x}(k)-s_{d}(k+1)\right. \\
\left.-D_{1}+\sum_{l=0}^{k}\left[s(l)-s_{d}(k)\right]\right\} .
\end{array}
$$

Theorem 2. If $g>2 D_{2} / \pi, \varepsilon>g \tan \left(D_{2} / g\right)$ and disturbance $D(k)$ satisfies (36), $k_{0} \leq k_{0 d}+2$ and (21) is satisfied for any $k \geq k_{0}$. Moreover, for any $k \geq \max \left\{k_{0}+1,2\right\}$, the absolute value of the plant's sliding variable is bounded by $\varepsilon+\Delta$.

Proof. First, we express $s(k+1)$ by considering (5) and (37) as

$$
\begin{aligned}
& \operatorname{sgn}[s(k+1)]|s(k+1)| \\
& =\operatorname{sgn}\left[s_{d}(k)\right]\left\{\left|s_{d}(k)\right|-g \arctan \left[\frac{\left|s_{d}(k)\right|}{g}\right]-\varepsilon\right\} \\
& \quad-D(k)+D_{1}-\sum_{l=0}^{k}\left[s(l)-s_{d}(l)\right] .
\end{aligned}
$$

Using 37 for any $k \geq 1$, the last element on the right-hand side of (39) can be expressed as

$$
\sum_{l=0}^{k}\left[s(l)-s_{d}(l)\right]=-D(k-1)+D_{1} .
$$

We substitute (40) into (39) and get

$$
\begin{aligned}
& \operatorname{sgn}[s(k+1)]|s(k+1)| \\
& =\operatorname{sgn}\left[s_{d}(k)\right]\left\{\left|s_{d}(k)\right|-g \arctan \left[\frac{\left|s_{d}(k)\right|}{g}\right]-\varepsilon\right\} \\
& \quad-D(k)+D(k+1) .
\end{aligned}
$$

Taking into account that, for any $k \geq k_{0 d}+1$, the desired sliding variable is upper bounded by $\varepsilon, \operatorname{sgn}\left[s_{d}(k-1)\right]=-\operatorname{sgn}\left[s_{d}(k)\right]$ and the disturbance satisfies (36), we conclude that choosing the control parameters as

$$
\begin{aligned}
& g>\frac{2 \Delta}{\pi}, \\
& \varepsilon>g \tan \left(\frac{\Delta}{g}\right)
\end{aligned}
$$

ensures the existence of the quasi-sliding mode for the plant for any $k \geq k_{0}=k_{0 d}+2$. Furthermore, from (37), for any $k \geq 2$, we may write

$$
s(k)=s_{d}(k)-D(k-1)+D(k-2) .
$$

From (17) and (36), for any $k \geq \max \left\{k_{0}+1,2\right\}$, the quasi-sliding mode band width is reduced to

$$
|s(k)| \leq \varepsilon+\Delta
$$

which completes the proof.

In the next step, following the same reasoning as in Section 2.3, the maximum value of the $i$-th state variable may be found. Assuming that the characteristic polynomial of the closed loop system satisfies (31) and considering (32) and (33), the absolute value of each state variable is bounded by

$$
\left|x_{i}(k)\right| \leq\left|(\varepsilon+\Delta) \boldsymbol{v}_{i} \sum_{l=0}^{n-1} \boldsymbol{A}_{\boldsymbol{c}}{ }^{l} \boldsymbol{b}(\boldsymbol{c} \boldsymbol{b})^{-1}\right|
$$

Moreover, the restriction of the rate of change in the reference sliding variable allows us to restrict the rate of change in the plant's sliding variable as well. According to the reaching law (37) we may express the change in $s(k)$ between successive steps as

$$
\begin{aligned}
& s(k)-s(k+1) \\
& =s_{d}(k)-D(k-1)+D_{1}-\sum_{l=0}^{k-1}\left[s(l)-s_{d}(l)\right] \\
& \quad-s_{d}(k+1)+D(k)-D_{1}+\sum_{l=0}^{k}\left[s(l)-s_{d}(l)\right] .
\end{aligned}
$$

Using (36) and (40), we obtain

$$
|s(k)-s(k+1)| \leq \varepsilon+g \frac{\pi}{2}+2 \Delta,
$$

which yields a smaller maximum rate of change than in (30). 


\section{Simulation results}

In this section we demonstrate the benefits of the reference trajectory based control strategy with two simulation examples. First, we shall compare the performance of our reference trajectory following strategy with the reaching law of Gao et al. (1995) and the inverse tangent reaching law (Leśniewski and Bartoszewicz, 2015). Next, we shall demonstrate the additional benefits of the modified strategy for slowly varying disturbance.

We consider a simple second-order continuous-time plant, consisting of an integrator and a first-order inertial element, discretized with the discretization period equal to 1 second. This kind of systems may, e.g., model mechanical actuators. The discrete time plant is described by

$\boldsymbol{x}(k+1)=\left[\begin{array}{ll}1 & 0.906 \\ 0 & 0.819\end{array}\right] \boldsymbol{x}(k)+\left[\begin{array}{c}0.468 \\ 0.906\end{array}\right][u(k)+d(k)]$.

The aim is to drive the system from the initial state $\boldsymbol{x}_{0}=\boldsymbol{x}(0)=\left[\begin{array}{ll}25 & 0\end{array}\right]^{T}$ to the desired state $\boldsymbol{x}_{d}=\left[\begin{array}{ll}0 & 0\end{array}\right]^{T}$. We select the sliding surface so that (31) holds. Therefore, the control vector $\boldsymbol{c}=\left[\begin{array}{ll}1 & 0.4833\end{array}\right]$. The disturbance $d(k)$ has the following values: $d(k)=-1$ for $k \in[1,20]$ and $d(k)=1$ for $k \in[21,40]$. Consequently, the mean disturbance $D_{1}=0$ and the maximum disturbance deviation $D_{2} \approx 0.906$.

We begin with computer simulations for the first three control methods. We choose the control parameters to ensure a comparable pace of convergence for all strategies. For Gao's reaching law, we chose $q=0.4$ and $\varepsilon=2.72$. For the strategy of Leśniewski and Bartoszewicz (2015), we set $g=4.6$ and $\varepsilon=0.87$, and for the trajectory following strategy, $g$ was set to 8 and $\varepsilon$ to 0.92 . This choice guarantees that in all the strategies the first change the sign of the sliding variable occurs between steps 2 and 3 . The results of our simulations are presented in Figs. 1-5. We plot the trajectories obtained with the trajectory following strategy with solid line, inverse tangent strategy with a dashed line, and Gao's strategy with a dotted line. Figures 1 and 2 show the evolution of the sliding variable of the plant during the whole control process and in the sliding phase only. All three control methods ensure the convergence of the system to the sliding plane in finite time and guarantee the sliding motion afterwards. As clearly visible, the reference trajectory following control method ensures a significant reduction in the width of the ultimate band.

Figure 3 presents the control signal. It is demonstrated that our new control method requires a smaller control effort in the sliding phase than the other strategies considered. We calculated the errors of the state variables in the quasi-steady state according to (32). The calculated maximum absolute error of the first state variable for the trajectory following method is

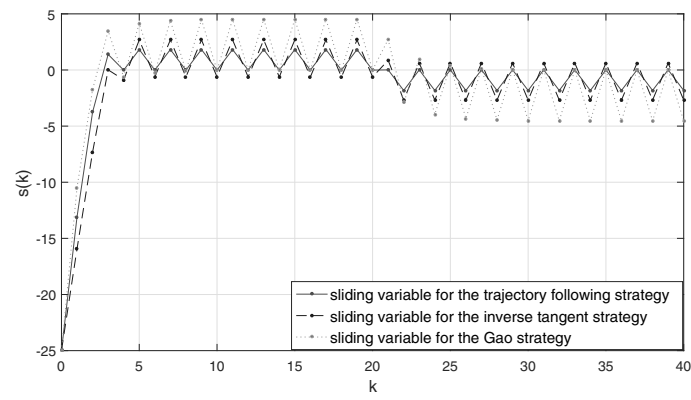

Fig. 1. Evolution of the sliding variable of the plant.

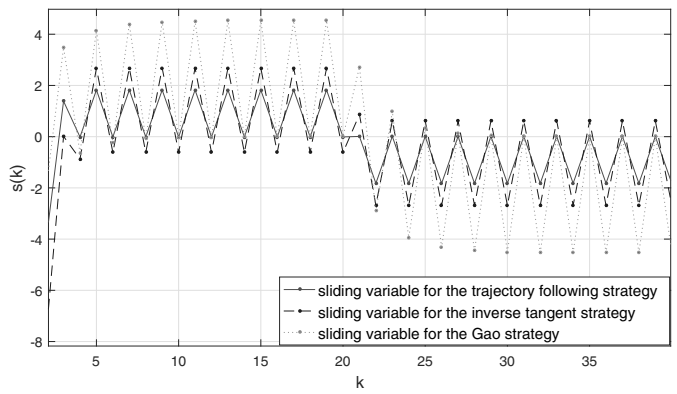

Fig. 2. Comparison of the ultimate bands.

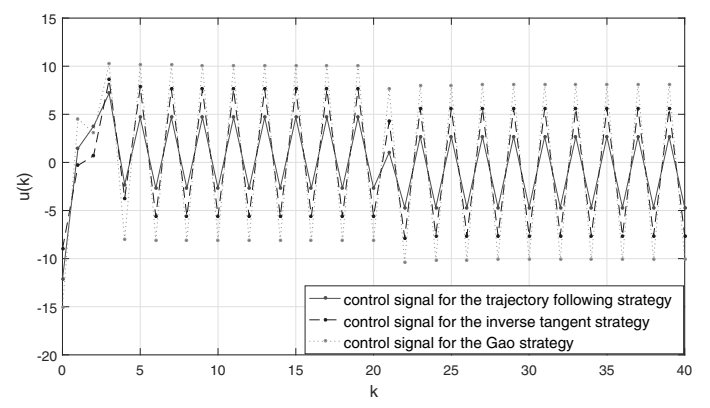

Fig. 3. Control signal for the plant.

$\left|x_{1}(k)\right|=0.94$, and that of the second state variable is $\left|x_{2}(k)\right|=1.83$, whereas for the inverse tangent strategy the absolute errors are $\left|x_{1}(k)\right|=1.15,\left|x_{2}(k)\right|=3.40$ and for the Gao's method they are $\left|x_{1}(k)\right|=2.34$, $\left|x_{2}(k)\right|=4.53$. All the computed error values may be verified in Figs. 4 and 5, which show the evolution of the state variables.

As one can see from the presented graphs, the reference trajectory based sliding mode control strategy ensures not only an improvement in the robustness of the system in comparison with the existing control methods, but also a significant reduction in the control effort.

Next, we shall present the additional benefits of the implementation of the modified strategy in comparison with the reference trajectory following 


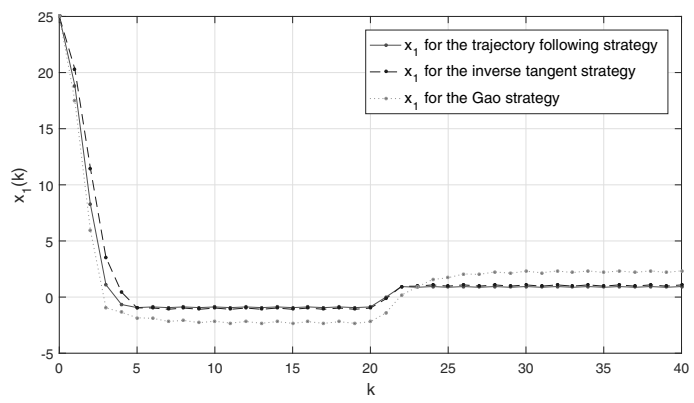

Fig. 4. First state variable $x_{1}$ of the plant.

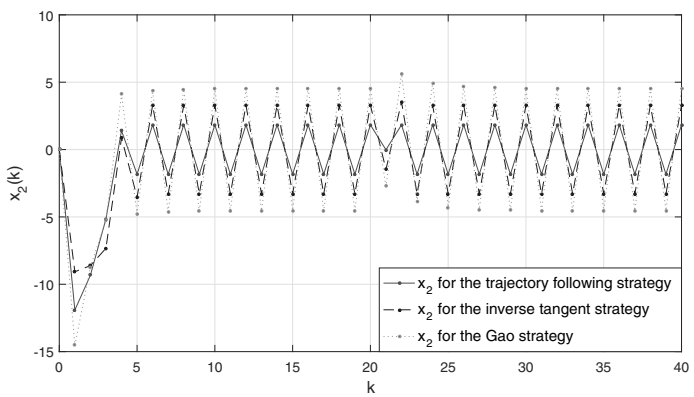

Fig. 5. Second state variable $x_{2}$ of the plant.

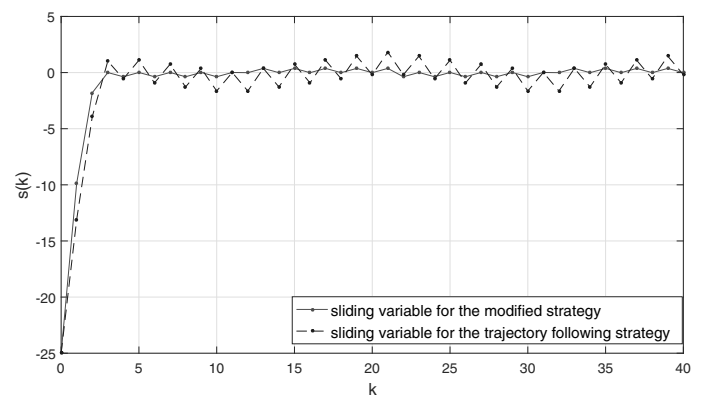

Fig. 6. Evolution of the sliding variable of the plant.

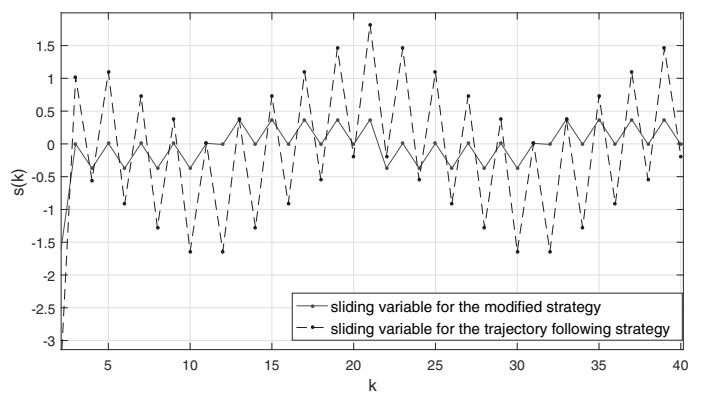

Fig. 7. Comparison of the ultimate bands.

\section{Conclusions}

This paper introduced a new, switching type reference trajectory based sliding mode control strategy for discrete time systems. First, the desired trajectory was obtained using the inverse tangent reaching law of Leśniewski and Bartoszewicz (2015). The reference trajectory does not depend on any perturbation. Next, we proposed a new trajectory following reaching law for the real disturbed system. We proved that an appropriate choice of the desired trajectory parameters ensures the existence of the quasi-sliding motion according to the fundamental definition by Gao et al. (1995). Moreover, we demonstrated that the implementation of our control strategy guarantees a significant reduction in the width of the ultimate band and maximum deviations of all state variables from their demand values in comparison with Gao's control method. Afterwards, we considered slowly varying disturbances and proposed a modification of our reaching law, which results in a further improvement in the robustness.

Lastly, the benefits of our two control strategies were verified with a simulation example. The performance of the reference trajectory based reaching law has been compared with the results of Gao's reaching law and the inverse tangent reaching law proposed by Leśniewski and Bartoszewicz (2015). Next, the benefits of the to $\left|x_{1}(k)\right|=0.19$ and $\left|x_{2}(k)\right|=0.74$. 


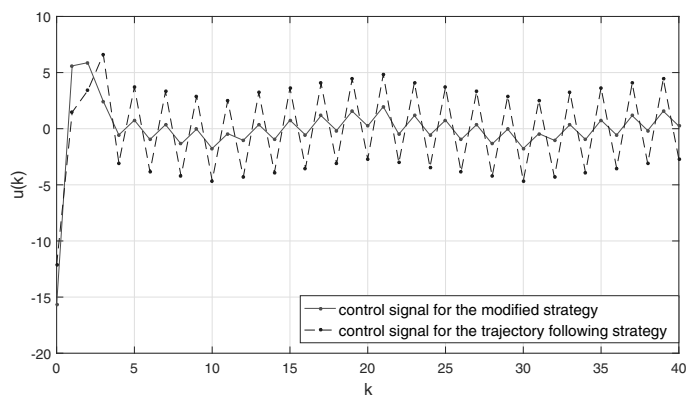

Fig. 8. Control signal for the plant.

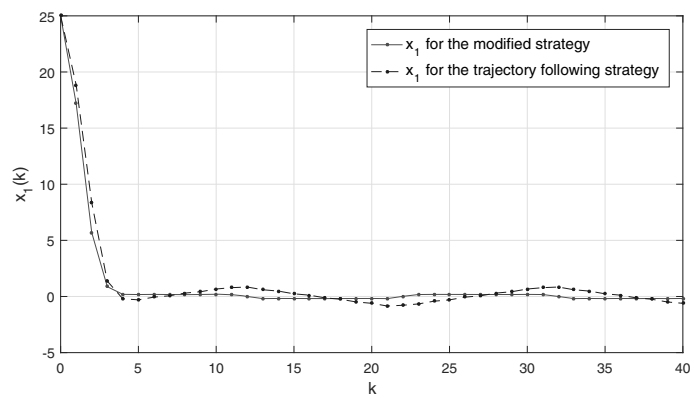

Fig. 9. First state variable $x_{1}$ of the plant.

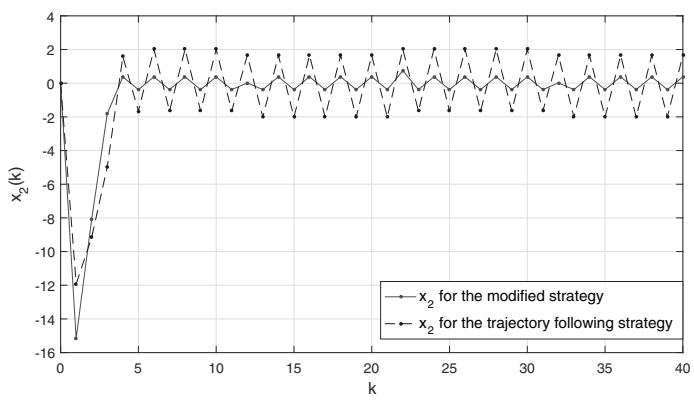

Fig. 10. Second state variable $x_{2}$ of the plant.

implementation of the modified strategy have been verified as well. The calculated errors of all state variables clearly show that both the trajectory following sliding mode control strategies ensure an improvement in the system's robustness without compromising the controller's efficiency.

\section{References}

Bartolini, G., Ferrara, A. and Usai, E. (1998). Chattering avoidance by second order sliding mode control, IEEE Transactions on Automatic Control 43(2): 241-246.
Bartolini, G., Ferrara, A., Usai, E. and Utkin, V. I. (2000). On multi-input chattering-free second-order sliding mode control, IEEE Transactions on Automatic Control 45(5): 1711-1717.

Bartoszewicz, A. (1996). Remarks on 'Discrete-time variable structure control systems', IEEE Transactions on Industrial Electronics 43(1): 235-238.

Bartoszewicz, A. (1998). Discrete-time quasi-sliding mode control strategies, IEEE Transactions on Industrial Electronics 45(4): 633-637.

Bartoszewicz, A. and Leśniewski, P. (2016). New switching and nonswitching type reaching laws for SMC of discrete time systems, IEEE Transactions on Control Systems Technology 24(2): 670-677.

Chakrabarty, S. and Bandyopadhyay, B. (2015). A generalized reaching law for discrete time sliding mode control, Automatica 52(1): 83-86.

Chakrabarty, S. and Bandyopadhyay, B. (2016). A generalized reaching law with different convergence rates, Automatica 63(1): 34-37.

Chakrabarty, S. and Bartoszewicz, A. (2016). Improved robustness and performance of discrete time sliding mode control systems, ISA Transactions on Industrial Informatics 65(1): 143-149.

Chang, F.J., Twu, S.H. and Chang, S. (1990). Adaptive chattering alleviation of variable structure systems, IEE Proceedings D: Control Theory and Applications 137(1): 31-39.

DeCarlo, R.A., Żak, S. and Matthews, G. (1988). Variable structure control of nonlinear multivariable systems: A tutorial, Proceedings of the IEEE 76(3): 212-232.

Draženović, B. (1969). The invariance conditions in variable structure systems, Automatica 5(3): 287-295.

Edwards, C. and Spurgeon, S. (1998). Sliding Mode Control: Theory and Applications, Taylor \& Francis, London.

Emelyanov, S.V. (1957). A method to obtain complex regulation laws using only the error signal or the regulated coordinate and its first derivatives, Avtomatika $i$ Telemekhanika 18(10): 873-885.

Emelyanov, S.V. and Utkin, V.I. (1964). Stability of motion of a class of variable structure control systems, Izvestiya $A N$ SSSR: Technical Cybernetics 2(1): 140-142.

Furuta, K. (1990). Sliding mode control of a discrete system, Systems \& Control Letters 14(2): 145-152.

Gao, W.B., Wang, Y. and Homaifa, A. (1995). Discrete-time variable structure control systems, IEEE Transactions on Industrial Electronics 42(2): 117-122.

Golo, G. and Milosavljević, C. (2000). Robust discrete-time chattering free sliding mode control, Systems \& Control Letters 41(1): 19-28.

Hung, J.Y., Gao, W.B. and Hung, J.C. (1993). Variable structure control: A survey, IEEE Transactions on Industrial Electronics 40(1): 2-22.

Itkis, U. (1976). Control Systems of Variable Structure, John Wiley \& Sons, New York, NY. 
Kotta, U., Sarpturk, S.Z. and Istefanopulos, Y. (1989). Comments on 'On the stability of discrete-time sliding mode control systems', IEEE Transactions on Automatic Control 34(9): 1021-1022.

Latosiński, P. (2017). Reaching law based discrete time switching quasi-sliding mode controller, Proceedings of 22nd International Conference on Methods and Models in Automation and Robotics, MMAR, Międzyzdroje, Poland, pp. 414-418.

Latosiński, P. and Bartoszewicz, A. (2018). Discrete time sliding mode controllers with relative degree one and two switching variables, Journal of the Franklin Institute 355(15): 6889-6903.

Leśniewski, P. and Bartoszewicz, A. (2015). Inverse tangent based switching type reaching law for discrete time sliding mode control systems, Proceedings of the European Control Conference, ECC'15, Linz, Austria, pp. 2390-2395.

Luis-Delgado, J.D., Al-Hadithi, B.M. and Jiménez, A. (2017). A novel method for the design of switching surfaces for discretized MIMO nonlinear systems, International Journal of Applied Mathematics and Computer Science 27(1): 5-17, DOI: 10.1515/amcs-2017-0001.

Ma, H., Wu, J. and Xiong, Z. (2017). A novel exponential reaching law of discrete-time sliding-mode control, IEEE Transactions on Industrial Electronics 64(5): 2245-2251.

Milosavljević, C. (1985). General conditions for the existence of a quasi-sliding mode on the switching hyperplane in discrete variable structure systems, Automation and Remote Control 46(3): 307-314.

Monsees, G. and Sherpen, J.M.A. (2001). Discrete-time sliding mode control with a disturbance estimator, Proceedings of the European Control Conference, ECC 2001, Porto, Portugal, pp. 3270-3275.

Niu, Y., Ho, D.W.C. and Wang, Z. (2010). Improved sliding mode control for discrete-time systems via reaching law, IET Proceedings on Control Theory \& Applications 4(11): 2245-2251.

Qu, S., Xia, X. and Zhang, J. (2014). Dynamics of discrete-time sliding-mode-control uncertain systems with a disturbance compensator, IEEE Transactions on Industrial Electronics 61(7): 3502-3510.

Ren, Y., Liu, Z., Liu, X. and Zhang, Y. (2013). A chattering free discrete-time global sliding mode controller for optoelectronic tracking system, Mathematical Problems in Engineering 2013(2): 1-8.

Sabanovic, A. (2011). Variable structure systems with sliding modes in motion control-a survey, IEEE Transactions on Industrial Informatics 7(2): 212-223.

Sarpturk, S.Z., Istefanopulos, Y. and Kaynak, O. (1987). On the stability of discrete-time sliding mode control systems, IEEE Transactions on Automatic Control 22(10): 930-932.
Utkin, V.I. (1977). Variable structure systems with sliding modes, IEEE Transactions on Automatic Control 22(2): 212-222.

Utkin, V.I. (1978). Sliding Modes and Their Applications in Variable Structure Systems, Mir Publishers, Moscow, pp. 212-222.

Utkin, V.I. (1984). Variable structure systems: Present and future, Automatic Remote Control 44(9): 1105-1220.

Utkin, V.I. and Drakunov, S.V. (1989). On discrete-time sliding modes, IFAC Proceedings Volumes 22(3): 273-278.

Veselic, B., Perunicic-Draženović, B. and Milosavljevic, C. (2010). Improved discrete-time sliding-mode position control using Euler velocity estimation, IEEE Transactions on Industrial Informatics 57(11): 3840-3847.

Vivekanandan, C., Prabhakar, R. and Gnanambigai, M. (2008). A redefined quasi-sliding mode control strategy, Proceedings of World Academy of Science 15(1): 292-295.

Zhang, F. (2016). Switching reaching law based switched sliding mode control, Proceedings of the Chineese Control Conference, CCC, Chengdu, China, pp. 4735-4739.

Zinober, S.I., Ei-Ghezawi, O.M.E. and Billings, S.A. (1982). Multivariable variable structure adaptive model following control systems, IEE Proceedings D: Control Theory and Applications 129(1): 6-12.

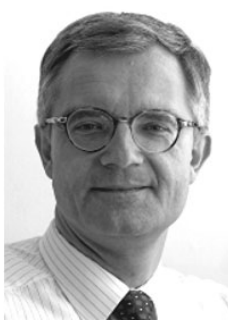

Andrzej Bartoszewicz received his $\mathrm{PhD}$ degree in 1993. He is currently a professor at the Łódz University of Technology, the head of the Electric Drive and Industrial Automation Unit and the director of the Institute of Automatic Control. He has authored three monographs and over 300 papers, primarily in the field of discrete time sliding mode control, inventory management and congestion control in data transmission networks. Professor Andrzej Bartoszewicz is a corresponding member of the Polish Academy of Sciences.

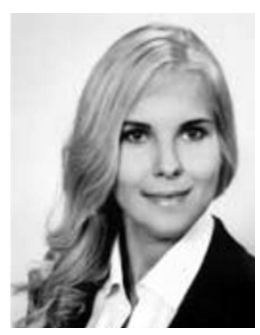

Katarzyna Adamiak received a BSc degree in mechatronics in 2017 and an MSc degree in control engineering in 2018. She is currently a $\mathrm{PhD}$ student at the Łódź University of Technology. Her main area of interest is discrete time sliding mode control.

Received: 4 December 2018

Revised: 15 March 2019

Accepted: 19 April 2019 